\title{
Análise do chá de hibisco da life natural
}

\author{
Dr. Fabiano de Abreu Agrela Rodrigues ${ }^{1}$ \\ deabreu.fabiano@gmail.com
}

Sandra Ribeiro ${ }^{2}$

\section{RESUMO}

O hibisco é uma planta da família Malvaceae. No Brasil, a Agência Nacional de Vigilância Sanitária (Anvisa), através da Portaria nº 519, de 1998, permite o uso das flores do hibisco para serem consumidas como chá, preparadas através de infusão ou decocção. O objetivo do presente estudo é avaliar os efeitos do produto chá de hibisco da empresa Life Natural ${ }^{\circledR}$. O presente estudo se trata de uma revisão de literatura realizada por meio das seguintes bases de dados: PubMed, Scielo e Google Acadêmico. É possível afirmar seus benefícios na redução da pressão arterial, colesterol LDL, gordura localizada, melhora dos sinais clínicos do envelhecimento e atuante como um complemento nos tratamentos de depressão.

Palavras-chave: hibisco; chá; extrato natural; colesterol LDL; pressão arterial

${ }^{1}$ Fabiano de Abreu Agrela Rodrigues - PhD, Neurocientista, biólogo, historiador, antropólogo com formações em neuropsicologia, psicologia e psicanálise.

${ }^{2}$ Sandra Ribeiro - Naturopata. 
Agrela Rodrigues y Sandra Ribeiro...

\title{
Life Natural's Hibiscus Tea Review
}

\begin{abstract}
Hibiscus is a plant in the Malvaceae family. In Brazil, the National Health Surveillance Agency (Anvisa), through Ordinance No. 519, of 1998, which allows the use of hibiscus flowers to be consumed as tea, prepared through infusion or decoction. Thus, the aim of this study and evaluation of the effects of the hibiscus tea product from the Life Natural ${ }^{\circledR}$ company. This study is a literature review carried out through the following databases: PubMed, Scielo and Google Scholar. Its benefits can be affirmed in reducing blood pressure, LDL cholesterol, localized reduction and improvement of aging and acting as an adjunct to treatments for depression.
\end{abstract}

Keywords: hibiscus; tea; natural extract; LDL cholesterol

Artículo recibido: 02 enero 2022 Aceptado para publicación: 28 enero 2022 Correspondencia: deabreu.fabiano@gmail.com Conflictos de Interés: Ninguna que declarar 


\section{INTRODUÇÃO}

\subsection{Hibísco}

A rosa-sinensis L. da família Malvaceae, popularmente conhecida como mimo-de-vênus ou hibisco-da-china, pampola, amor-de-homens, aurora, pampulha ou hibisco. Reportadas nos livros de plantas medicinais e culinária (Izquierdo-Vega, 2020).

É um arbusto de ciclo anual, envolvendo diversas espécies podendo atingir cerca de $3 \mathrm{~m}$ de altura. Suas flores são simples ou dobradas, durando cerca de um ou dois dias. Suas pétalas possuem um leve gosto cítrico, sendo comumente utilizadas em saladas, infusão, geleias, licores, dentre outros (Izquierdo-Vega, 2020).

Cultivado em regiões tropicais e subtropicais. A corola é constituída por cinco sépalas com coloração vermelha em forma de cone formando um cálice. Na base do cálice, está localizado o calículo ou pequeno cálice disposto em círculo. Em sua cápsula deiscente é encontrado o fruto que contém aspecto aveludado com média de $2 \mathrm{~cm}$ de comprimento, envolvendo as sementes (Jeffery, 2020).

Alimento funcional, nos países da Ásia (Japão, China, Coreia e Taiwan) onde são utilizados seus cálices desidratados para a produção de bebidas, alimentos, conservantes e antioxidantes (Jeffery, 2020). No Brasil, a Agência Nacional de Vigilância Sanitária (Anvisa), através da Portaria $n^{\circ} 519$, de 1998, que permite o uso das flores do hibisco serem consumidas como chá, preparadas através de infusão ou decocção (Jeffery, 2020).

\subsection{Benefícios para a saúde}

Devido a suas altas concentrações de antocianinas, vitamina C e polifenóis, substâncias com alta atividade antioxidante, combatem os radicais livres, moléculas liberadas pelo metabolismo, a partir de elétrons instáveis e reativos, envolvidos nas doenças degenerativas de envelhecimento e morte celular gerando assim, o envelhecimento precoce (Sim, 2021).

A partir de um estudo onde foi avaliado a atividade antioxidante do hibisco, a partir do espectrofotométrico a $518 \mathrm{~nm}$, demonstrou elevadas concentrações de compostos fenólicos, altamente antioxidantes (Sobota, 2016).

A pressão arterial refere-se à pressão realizado pelo sangue contra a parede das artérias. Quando esta pressão aumenta é chamada hipertensão arterial e quando diminui, hipotensão arterial (Parati, 2018). 
Mckay et. al. 2010, avaliou os efeitos do hibisco em relação a diminuição da pressão arterial, a partir de um ensaio clínico randomizado controlado e duplamente cego, com 65 adultos hipertensos. Sendo assim, foi constatado que o grupo que realizou a ingestão diário de chá de hibisco apresentou diminuição na pressão arterial, sendo benéfico para esta população.

Corroborando com os resultados, outro estudo também avaliou a atividade do chá de hibisco em 46 indivíduos hipertensos. Esses indivíduos faziam a ingestão diária de 2 xícaras de chá durante o período de um mês, demonstrando diminuição significativa da pressão arterial sistólica dos participantes (Jalalyazdi, 2019).

A lipoproteína de baixa densidade (colesterol LDL) é da família das lipoproteínas, popularmente conhecido como colesterol ruim, pois se acumula nas paredes dos vasos sanguíneos, quando encontrado em altas taxas aumenta as chances do desenvolvimento de patologias como; aterosclerose, ataque cardíaco e Acidente Vascular Cerebral (AVC). Em um estudo experimental, com 30 ratos albinos saudáveis divididos em dois grupos, o primeiro que recebeu $1,5 \mathrm{mg} / \mathrm{kg}$ e o segundo recebeu $200 \mathrm{mg} / \mathrm{kg}$, ao longo de um mês, demonstrando que no primeiro grupo houve diminuição significativa dos níveis de colesterol LDL e triglicerídeos (Olatunji, 2005).

Outro estudo pré-clínico avaliou os efeitos do hibisco em coelhos com aterosclerose grave na aorta, demonstrando que após 10 semanas de intervenção, a partir das análises histopatológicas, demonstrou redução da formação de células espumosas e inibiu a migração de células de músculo liso e calcificação no vaso sanguíneo de coelhos, demonstrando assim que houve redução do perfil dos lipídios séricos (Chen, 2003).

O ganho de peso é algo que afeta a autoestima de diversas pessoas, tanto homens quanto mulheres. Seu aumento pode trazer malefícios a saúde, como: doenças cardíacas coronarianas, hipertensão, diabetes, osteoartrite e certos tipos de câncer (Almeida, 2020). Em um ensaio clínico duplo-cego, randomizado, controlado por placebo, com um total de 84 indivíduos, sem comorbidades e índice de massa corporal (IMC) entre 25 e 35 $\mathrm{kg} / \mathrm{m}^{2}$ (Marhuenda, 2020), foi demonstrado que os indivíduos tratados com uma dieta rica em hibisco apresentaram redução no peso corporal. O índice de massa corporal e a massa gorda medida tanta pela bioimpedância quanto pela densitometria (Marhuenda, 2020). Por meio de outro estudo similar com 30 indivíduos adultos, também foi possível constatar que o grupo que recebeu a intervenção de duas doses de chá, todos os dias, 
apresentou diminuição do peso corporal, bem como no IMC (Prieto, 2013).

Para avaliação dos efeitos diuréticos, a partir de um estudo experimental com amostra de 20 ratos wistar, que obtiveram suas amostras avaliadas após 18 horas, para avaliação de volume medido e concentração de eletrólito. A partir dos resultados obtidos foi possível relatar os efeitos benéficos do uso do hibisco para fins diuréticos (Njinga, 2020).

Também podem atuar positivamente nos neurotransmissores dopamina e serotonina. Ambos atuam em diversas áreas do corpo, a partir do sistema nervoso central (SNC).

A dopamina é responsável pela transmissão dos impulsos nervosos entre um neurônio e outro. Além de atuar nas emoções, aprendizado, humor e atenção, quando o indivíduo possuí baixos níveis de sua produção é possível que desenvolva depressão.

A serotonina também pode ser encontrada no sistema nervoso, digestivo e plaquetas. Atua na regulagem do humor, sono, apetite, ritmo cardíaco, temperatura corporal, sensibilidade, bem como nas funções cognitivas. Por este motivo quando o indivíduo possuí baixos níveis, pode causar mau humor, dificuldade para dormir, ansiedade ou depressão.

Shewale et. al. 2012, realizou um ensaio pré clinico, onde os animais com depressão, foram submetidos à ingestão de hibisco, demonstrando uma melhora nos níveis de depressão destes animais.

Outro estudo avaliou os efeitos do chá de hibisco em modelo de animais com a doença de Parkinson, demonstrando potente atividade antioxidante e eliminadora dos radicais livres. Também houve diminuição significativa em relação ao comportamento de ansiedade e inibição da depressão. No teste de labirinto em cruz elevado e testes de natação forçada, houve um ótimo desempenho da memória espacial em tarefas de labirinto em Y e labirinto de braço radial foi melhorado, sugerindo efeitos positivos na formação da memória (Foyet, 2011).

\subsection{Chá solúvel hibisco da Life Natural}

O produto chá de hibisco da empresa Life Natural ${ }^{\circledR}$ utiliza componentes naturais como comprovado segundo dados e registros enviados.

Atuando na melhora do envelhecimento cutâneo por ser rico em antioxidante e vitaminas. Diminuindo a pressão arterial, colesterol LDL e reduzindo o peso corporal. Auxiliando assim em doenças relacionadas à obesidade como; infarto, AVC, aterosclerose dentre outras. 
Para obter um bom resultado, o fabricante recomenda a ingestão de 2 copos de $200 \mathrm{ml}$ por dia. A seguir a bula do chá de hibisco com todas as informações passadas pelo fabricante:

\begin{tabular}{|l|c|c|}
\hline TABELA NUTRICIONAL & QTD. /DOSE & \%VD* \\
\hline VALOR ENERGÉTICO & $32 \mathrm{kcal}$ & $2 \%$ \\
\hline Carboidratos & $8 \mathrm{~g}$ & $3 \%$ \\
\hline Proteínas & $1,7 \mathrm{~g}$ & $3 \%$ \\
\hline
\end{tabular}

*Não contém quantidades significativas de Gorduras Totais, Gorduras Saturadas, Gorduras Trans, Fibra Alimentar e Sódio.

O fabricante sugere, utiliza-lo desta maneira: Adicione 1 colher de sopa (10g) de Chá de Hibisco com Colágeno em 1 copo de $200 \mathrm{ml}$ de água fria ou quente, misture bem e tome.Sendo indicado para indivíduos maiores de 19 anos e contraindicado, para gestantes, lactantes e devido conter corante em sua formula é contraindicado para alérgicos.

\section{OBJETIVO}

Avaliar os efeitos do produto chá de hibisco da empresa Life Natural ${ }^{\circledR}$.

\section{MÉTODOS}

O presente estudo se trata de uma revisão de literatura realizada por meio das seguintes bases de dados: PubMed, Scielo e Google Acadêmico por meio das seguintes palavras chaves: hibisco, chá, extrato natural, colesterol LDL, pressão arterial e em inglês: hibiscus, tea, natural extract, LDL cholesterol. Bem como a bula do produto chá solúvel hibisco e através das informações passadas pelo fabricante do mesmo: Life Natural ${ }^{\circledR}$.

\section{CONSIDERAÇÕES FINAIS}

Através da análise da composição do produto, usando como referência, os dados enviados pela empresa Life Natural e ao entrevistar consumidores, a conclusão é de que os efeitos são favoráveis e de acordo com os estudos apresentados neste artigo.

Segundo o CEO da empresa "A verdade é que o chá de hibisco é indicado para todas as pessoas que desejam ter qualidade de vida. Na Life Natural, por exemplo, oferecemos o chá solúvel de hibisco com colágeno hidrolisado que, além de favorecer a perda de peso saudável, contribui para o bem-estar do indivíduo".

É possível afirmar a eficácia do produto de chá de hibisco já que contém substâncias que possuem comprovação científica por meio da publicação na revista científica e no Centro 
de Pesquisas e Análises Heráclito - CPAH Scientific Journal of Health confirmando que são eficientes para a redução da pressão arterial, colesterol LDL, gordura localizada, melhora do envelhecimento e atuante como um complemento nos tratamentos de depressão.

\section{REFERÊECIAS}

ALMEIDA, N. S. de; NOGUEIRA, A. M.; BONATO, G. S. L.; ARAUJO, B. C. G. de; TOLEDO, A. C. C. G. Effects of the application of pulsed combination therapy associated with medium frequency excitomotor current under the reduction of abdominal localized fat in women. Research, Society and Development, [S. l.], v. 9, n. 11, p. e36691110086, 2020. DOI: 10.33448/rsd-v9i11.10086.

CHEN, C.C., Hsu, J.D., Wang, S.F., Chiang, H.C., Yang, M.Y., Kao, E.S., Ho, Y.C., Wang, C.J. Hibiscus sabdariffa extract inhibits the development of atherosclerosis in cholesterol-fed rabbits. J Agric Food Chem. v. 27, n. 18, págs. 5472-7, 2003 doi: $10.1021 / \mathrm{jf030065w.}$

Foyet HS, Hritcu L, Ciobica A, Stefan M, Kamtchouing P, Cojocaru D. Methanolic extract of Hibiscus asper leaves improves spatial memory deficits in the 6hydroxydopamine-lesion rodent model of Parkinson's disease. J Ethnopharmacol. n. 27, v. 2, págs. 773-779, 2011 doi: 10.1016/j.jep.2010.11.011.

IZQUIERDO-VEGA, J.A., Arteaga-Badillo, D.A., Sánchez-Gutiérrez, M., MoralesGonzález, J.A., Vargas-Mendoza, N., Gómez-Aldapa, C.A., Castro-Rosas, J., Delgado-Olivares, L., Madrigal-Bujaidar, E., Madrigal-Santillán, E. Organic Acids from Roselle (Hibiscus sabdariffa L.) A Brief Review of Its Pharmacological Effects. Biomedicines. n.28, v. 5, pág. 100, 2020 doi: 10.3390/biomedicines8050100.

JALALYAZDI, M., Ramezani, J., Izadi-Moud, A., Madani-Sani, F., Shahlaei, S., Ghiasi, S.S. Effect of hibiscus sabdariffa on blood pressure in patients with stage 1 hypertension. J Adv Pharm Technol Res. n. 10, v. 3, págs. 107-111, 2019 doi: 10.4103/japtr.JAPTR_402_18

JEFFERY, T.D., Richardson, M.L. A review of the effectiveness of hibiscus for treatment of metabolic syndrome. J Ethnopharmacol. n. 24, v. 270, e113762, 2021 doi: 10.1016/j.jep.2020.113762.

MARHUENDA, J., Perez, S., Victoria-Montesinos, D., Um ensaio randomizado, duplo- 
cego e controlado por placebo para determinar a eficácia de um extrato polifenólico ( Hibiscus sabdariffa e Lippia citriodora) na redução da massa gorda corporal em indivíduos saudáveis. Alimentos. n. 9, v. 3, págs. 55, 2020

MCKAY, D.L., Chen, C.Y., Saltzman, E., Blumberg, J.B. Hibiscus sabdariffa L. tea (tisane) lowers blood pressure in prehypertensive and mildly hypertensive adults. J Nutr. 2010 n. 140, v. 2, págs. 298-303, 2010 doi: 10.3945/jn.109.115097.

NJINGA, N.S., Kola-Mustapha, A.T., Quadri, A.L., Atolani, O., Ayanniyi, R.O., Buhari, M.O., Toxicity assessment of sub-acute and sub-chronic oral administration and diuretic potential of aqueous extract of Hibiscus sabdariffa calyces. Heliyon. n. 21, v. 9, e04853, 2020 doi: 10.1016/j.heliyon.2020.e04853

OLATUNJI, L.A., Adebayo, J.O., Oguntoye, O.B., Olatunde, N.O., Olatunji, V.A., Soladoye, A.O. Effects of aqueous extracts of petals of red and greenHibiscus sabdariffa on plasma lipid and hematological variables in rats. Pharm. Biol. N. 43, págs. 471-474, 2005

PARATI, G., Stergiou, G.S., Dolan, E., Bilo, G. Blood pressure variability: clinical relevance and application. J Clin Hypertens (Greenwich). 2018 n. 20, v. 7, págs. 1133-1137, 2018 doi: 10.1111/jch.13304.

PRIETO, D., Anez, R., Rojas, J., Bermudez, V., Efeito da administração de uma infusão aquosa de Hibiscus Sabdariffa L. no índice de massa corporal, apetite e saciedade em indivíduos com obesidade grau I. AVFT [online]. v .32, n.3, págs.34-40, 2013

SHEWALE, P.B., Patil, R.A., Hiray, Y.A. Antidepressant-like activity of anthocyanidins from Hibiscus rosa-sinensis flowers in tail suspension test and forced swim test. Indian J Pharmacol. n. 44, v. 4, págs. 454-457, 2012 doi: 10.4103/02537613.99303.

SIM, Y.Y., Nyam, K.L. Hibiscus cannabinus L. (kenaf) studies: Nutritional composition, phytochemistry, pharmacology, and potential applications. Food Chem. n. 15, v. 344, e128582, 2021 doi: 10.1016/j.foodchem.2020.128582.

SOBOTA, J.F., Pinho, M.G., Oliveira, V.B., Perfil físico-químico e atividade antioxidante do cálice da espécie Hibiscus sabdariffa L. a partir do extrato aquoso e alcoólico obtidos por infusão e decocto. Revista Fitos, Rio de Janeiro, v.10, n. 1, págs. 91-93, 2016 